\title{
Impact of semiconductor quantum dots bandgap on reabsorption in luminescent concentrator
}

\author{
A.I. Shkrebtii ${ }^{1}$, A.V. Sachenko ${ }^{2}$, I.O. Sokolovskyi' ${ }^{2}$, V.P. Kostylyov ${ }^{2}$, M.R. Kulish ${ }^{2}$ \\ ${ }^{1}$ University of Ontario Institute of Technology, \\ 2000 Simcoe St. N., Oshawa, Ontario, L1H 7K4, Canada \\ ${ }^{2}$ V. Lashkaryov Institute of Semiconductor Physics, NAS of Ukraine, \\ 45, prospect Nauky, 03680 Kyiv, Ukraine \\ E-mail:sach@isp.kiev.ua
}

\begin{abstract}
We have investigated the influence of the average radius $\bar{r}$ and its dispersion $\Delta \vec{r}$ of the semiconductor quantum dots (QDs) used in luminescent solar concentrators (LSCs) on reabsorption. To minimize the detrimental reabsorption losses in LSCs, six semiconductors used to fabricate QDs with a wide range of their bulk band gap $E_{g 0}$ have been considered, specifically: $\mathrm{CdS}\left(E_{g 0}=2.42 \mathrm{eV}\right), \mathrm{CdSe},\left(E_{g 0}=1.67 \mathrm{eV}\right), \operatorname{CdTe}\left(E_{g 0}=\right.$ $1.5 \mathrm{eV}), \operatorname{InP}\left(E_{g 0}=1.27 \mathrm{eV}\right)$, InAs $\left(E_{g 0}=0.355 \mathrm{eV}\right)$, and PbSe $\left(E_{g 0}=0.27 \mathrm{eV}\right)$. Altering $\bar{r}$ and $\Delta \vec{r}$, we can determine the optimal size $\bar{r}$ for minimal reabsorption. As it was shown, decreasing the semiconductor bulk band gap from 2.42 down to $1.24 \mathrm{eV}$ we can get such optimum QD size $\bar{r}$ that reabsorption reduces even below the combined experimental error in determination of the absorption coefficient and luminescence intensity. Further reduction of the gap $E_{g 0}$, however, increases reabsorption at any values of $r$ and $\Delta r$ : for instance, for $\mathrm{PbSe}-$ based QDs of 1-nm radius and dispersion of 1\%, reabsorption reaches $54 \%$.
\end{abstract}

Keywords: photoluminescence, quantum dots, luminophor, reabsorption, solar panels, efficiency, concentrator.

doi: https://doi.org/10.15407/spqeo21.01.058

PACS 88.40.jm, 88.40.jp

Manuscript received 07.02.18; revised version received 09.03.18; accepted for publication 29.03.18; published online 29.03.18.

\section{Introduction}

Currently, intensive search for alternative approaches to reduce the solar energy cost is going. One of these approaches is to use luminescent solar concentrators (LSC). The luminescent solar concentrator is a transparent plate of organic or inorganic material that is doped by luminophor, and to the ends of which solar cells are attached. Among all types of luminophors, the use of semiconductor quantum dots (QDs) is the most attractive variant. QD diameter can vary from 1 up to $20 \mathrm{~nm}[1,2]$. QDs transform broad spectral range of light quanta in a narrow band. By changing the optical gap, radius $\bar{r}$ and dispersion $\Delta \vec{r}$, we can achieve the good match between spectral position of the luminescence band and the photoconverter's maximum sensitivity region.

Electrons and holes in QDs can be considered as quasi-particles in a three-dimensional (3D) potential box.
Quasi-particle's energy is counted from the ground potential (position of hole and electron quantum levels counted from the bottom of the conduction band and the top of the valence band, respectively). The energy position of quantum levels in the QD can be calculated using the formula $[2,3]$

$$
E_{n, l}^{e, h}=E_{g 0}+\frac{\hbar^{2} \varphi_{l, n}^{2}}{2 r^{2}}\left[\frac{1}{m_{e}}+\frac{1}{m_{h}}\right]-\frac{1.786 e^{2}}{4 \pi \varepsilon \varepsilon_{0} r}-0.248 E_{\mathrm{Ry}}^{*},
$$

where $E_{g 0}$ is the band gap of bulk semiconductor, $E_{\mathrm{Ry}}^{*}-$ Rydberg energy, $\varepsilon$ and $\varepsilon_{0}$ are the relative dielectric constants for semiconductor and its absolute value in vacuum, respectively, $\hbar$ - reduced Planck's constant, $m_{e}$ and $m_{h}$ - effective masses of the electron and hole, respectively, $e$ - electron charge, $r$ - nanocrystal core radius, $n=1,2,3, \ldots-$ principal quantum numbers, $l=$ $0,1,2, \ldots$ - orbital quantum numbers, while $\varphi_{l, n}-$ universal set of numbers [4], which are given in Table. 
Table. Values of the parameter $\varphi_{l, n}$ used in Eq. (1) [4].

\begin{tabular}{|l|c|c|c|c|}
\hline$l / n$ & 1 & 2 & 3 & 4 \\
\hline 0 & 3.142 & 6.263 & 9.425 & 12.566 \\
\hline 1 & 4.493 & 7.725 & 10.904 & 14.066 \\
\hline 2 & 5.764 & 9.095 & 12.323 & \\
\hline 3 & 6.988 & 10.417 & 13.698 & \\
\hline 4 & 8.183 & 11.705 & & \\
\hline 5 & 9.356 & 12.967 & & \\
\hline 6 & 10.513 & 14.207 & & \\
\hline 7 & 11.657 & & & \\
\hline 8 & 12.791 & & & \\
\hline 9 & 13.916 & & & \\
\hline
\end{tabular}

The first term in Eq. (1) is the semiconductor bulk bandgap $E_{g 0}$; the second term describes the energy $E_{l, n}^{e, h}$ of quantized levels due to carriers' confinement in the nanocrystal; the third term describes the energy reduction due to interaction of electrons and holes. The latter fourth term is the Rydberg energy, which does not depend on the size of the nanoparticles and can be usually neglected, except the cases of semiconductors with a low dielectric constant.

The QD absorption spectrum is formed by transitions between the size-quantized electron and hole levels with the same quantum numbers $n$ and $l$ (see Fig. 1a). In real nanocrystals, two main mechanisms are responsible for the broad absorption band: (i) at the nonzero absolute temperature there always is the thermal broadening of the quantized energy levels due to atomic vibrations and (ii) the nanocrystal size dispersion leads to the additional inhomogeneous broadening, which is temperature independent (Fig. 1b).

When calculating QD absorption spectra, the thermal energy level broadening is usually neglected, and the only contribution from nanocrystal size dispersion that is considered. In this case, the absorption spectrum can be calculated using the formula [5]:

$$
\begin{aligned}
& \alpha\left(E_{p h}\right)=10^{4} \frac{2 \pi \hbar e^{2}}{m_{0}^{2} c n_{r} \varepsilon_{0}} \frac{1}{V_{Q D}} \times \\
& \sum_{i} \frac{\left|P_{i}\right|^{2}}{3 E_{p h} \sqrt{2 \pi} \Delta E_{i}} \exp \left(-\frac{\left(E_{p h}-E_{i}\right)^{2}}{2 \Delta E_{i}^{2}}\right),
\end{aligned}
$$

where $E_{p h}$ is the photon energy, $m_{0}$ - mass of free electron, $c$ - speed of light in vacuum, $n_{r}-$ real part of the refractive index of bulk semiconductor, $V_{Q D}$ - volume of the quantum dot, $\Delta E_{i}$ - line width of the optical transition, $P_{i}$ and $E_{i}$ are the matrix element of momentum and energy with respect to each of the $i$-th exciton transition.

Eq. (2) was used in [5] to calculate the theoretical dependence of the absorpion spectra of CdSe QDs with their diameter ranging from 1.5 to $8 \mathrm{~nm}$, reaching good agreement of the calculated and experimental absorption spectra.

When being illuminated by sunlight, electrons are excited from the quantized levels of the valence band to the corresponding quantum energy levels of the conduction band. Both electrons and holes relax quickly (within a picosecond time interval) from high levels to corresponding lowest levels $E_{01}^{e}$ and $E_{01}^{h}$, respectively. Then, electrons recombine with holes as a result of the transition from $E_{01}^{e}$ to $E_{01}^{h}$, emitting the luminescent photon.

The majority of the researches on the nanocrystals properties consider an ensemble of QDs with $10 \%$ size dispersion (see, e.g., [6]) with the Gaussian distribution [7] of their radius $r$ around its mean value:

$P(r, \bar{r})=\frac{1}{\sqrt{2 \pi} \sigma_{r}} e^{-(r-\bar{r})^{2} / 2 \sigma_{r}^{2}}$.

In (3), $\bar{r}$ is the mean QD radius, the standard deviation $\sigma_{r}$ can be determined using electron microscopy data.

Each QD emits luminescence photons with its characteristic energy $h v_{i}$, the combined emission of the set of QDs forms a luminescent band. The intensity of luminescence band inherent to QDs $I_{P L}^{E n}$ is a sum of luminescence intensities $I_{P L}\left(h v_{i}, r_{i}\right)$, where $r_{i}$ is the variable radius of $i$-th $\mathrm{QD}$, emitting photons of $h \mathrm{v}_{i}$ energy ( $r_{i}$ is changed within the spread in the QD size). Therefore, following [7], the intensity of the luminescence band of the QD set can be described as:

$I_{P L}^{E n}=\sum_{i} \alpha\left(h v_{e x}, r\right) I_{P L}\left(h v_{i}, r_{i}\right) P\left(r_{i}, \bar{r}\right)$.

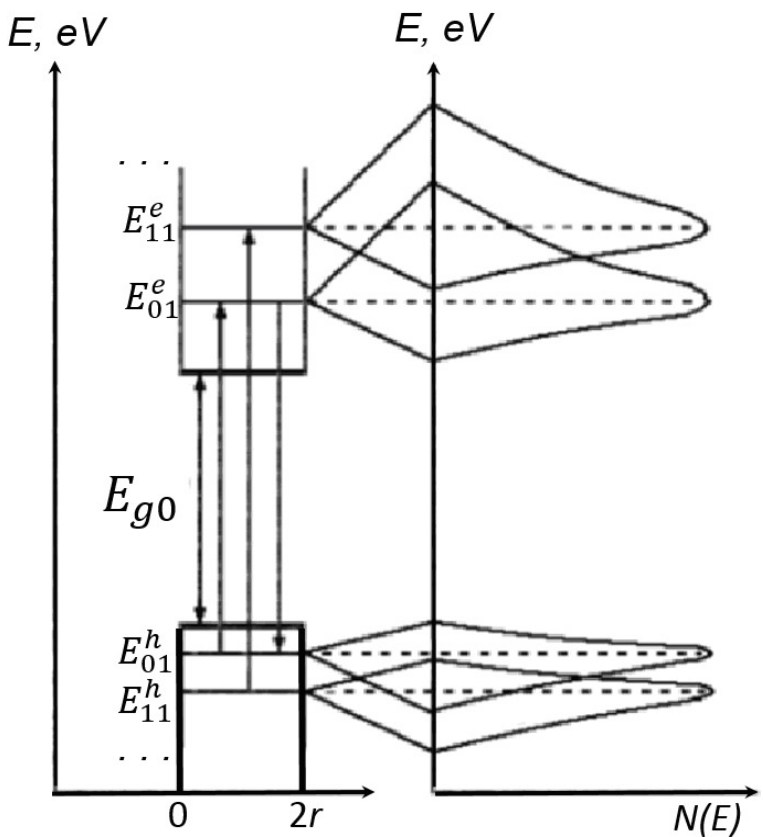

(a)

(b)

Fig. 1. Quantized energy levels of electrons and holes in QDs in the case of (a) 3D confined nanoparticle with its $2 r$ core size fixed and discrete energy levels $E_{n, l}^{e, h}$ (see the text for explanation). In the case (b), the QD size dispersion is present with the thermal broadening of the energy levels included. $E_{g 0}$ is the bulk semiconductor gap, and $N(E)$ is the carriers' density of states. 
The energy difference between the first maximum of the absorption band and the luminescence band maximum of QDs can be found as:

$\Delta h v=\frac{1.786 e^{2}}{4 \pi \varepsilon_{0} \varepsilon_{\infty} \vec{r}}-0.248 E_{\mathrm{Ry}}^{*}$,

where $\bar{r}$ is the average radius of the ensemble of nanoparticles. As it was found [7], the intensity of luminescence band, calculated using Eq. (4), correctly describes the corresponding experimental dependence for the ensemble of InP quantum dots.

Usually, a set of quantum dots contains particles of different, statistically distributed sizes, which results in both absorption and of luminescence spectra, dependent on characteristics of the QD ensemble. In particular, the luminescence band halfwidth increases with the quantum dots dispersion increase. Additionally, the thermal smearing of QDs energy levels results in increase of the absorption and luminescence bandwidth. As a result of the effect of thermal widening the energy levels and spread of quantum dots in size, the luminescence and absorption bands overlap, determining the value of reabsorption (reabsorption of luminescence quantum). The larger the spread of quantum dots, the greater the reabsorption value.

While the above expressions essentially correctly describe the evolution of luminescence and absorption spectra of the size dispersed QDs, they do not allow estimating the evolution of reabsorption with their radius. The purpose of this work is firstly to derive expressions that properly take into account the distribution of nanoparticle size and the thermal spreading the quantized levels on reabsorption. Second analysis of the derived expressions brings understanding the impact of the nanoparticle size and their dispersion of on absorption, luminescence and reabsorption.

\section{Absorption and luminescence spectra of the quantum dot core}

Usually, QDs with high luminescence quantum yield contain a core surrounded by one or more shells of semiconductor materials and a shell of organic material (Fig. 2). Typically, the core is made of narrow-gap semiconductor, which transforms the broad solar spectrum into a narrow luminescence band. The first (inorganic) shell ensures the dangling bonds passivation at the surface of the QD core. The band gap of this inorganic shell is wider than the core gap. If several inorganic shells are present, each successive shell differs from the previous by an increased band gap. The main purpose of the multi-shell design is to align semiconductor lattice parameter, reducing dislocations appearance. The outer organic shell consists of organic molecules, deposited on the top inorganic shell, and its main purpose is to prevent aggregation of QDs.

Geometric characteristics of QDs and their size dispersion are usually determined from the electron microscopy measurements: the average core size can be extracted from the absorption and luminescence spectra. To investigate evolution of reabsorption when QD dimensions are changed, it is sufficient to determine the average QD core size (radius or diameter). It can be done by comparing a known experimental position of the absorption spectrum maximum formed by electron transitions from the energy levels and with the theoretical one. Therefore, to extract the average QD radius we derived an expression for the quantum dot light absorption dependence on the photon energy $E_{p h}$ in the form:

$$
\alpha_{1}\left(E_{p h}\right)=A \int_{0}^{\infty} \frac{1}{r^{3}} \exp \left(\frac{\left(E_{p h}-E_{1}(r)\right)^{2}}{2 \sigma_{E}^{2}}\right) \exp \left(\frac{(r-\bar{r})^{2}}{2 \sigma_{r}^{2}}\right) d r,(6)
$$

Here, $A$ is the constant, which is QD radius, independent and determined from normalization of the absorption to unity. The first exponent (Gaussian distribution on the energy $E$ ) defines the thermal smearing $\sigma_{E}$ of the quantized energy levels in the conduction band and of the light holes valence band. When calculating the absorption spectrum from Eq. (6), it is sufficiently accurately to use $\sigma_{E}=2 k T$ ( $k$ is the Boltzmann constant, and $T$ - absolute temperature). The second exponential (Gaussian QD size distribution) relates QDs radius dispersion $\sigma_{r}$ and the optical spectra broadening. Here, the energy $E_{1}\left(r_{i}\right)$ corresponds to the absorption maximum of QD with its core radius $r_{i}$. Next, we calculate the shape of the absorption band formed by the electron transition from the level $E_{01}^{e}$ to $E_{01}^{h}$ one.

The energy spectrum of luminescence peak position is estimated using the formula (5). We derived the expression for a shape of the luminescence spectrum, which can be written as:

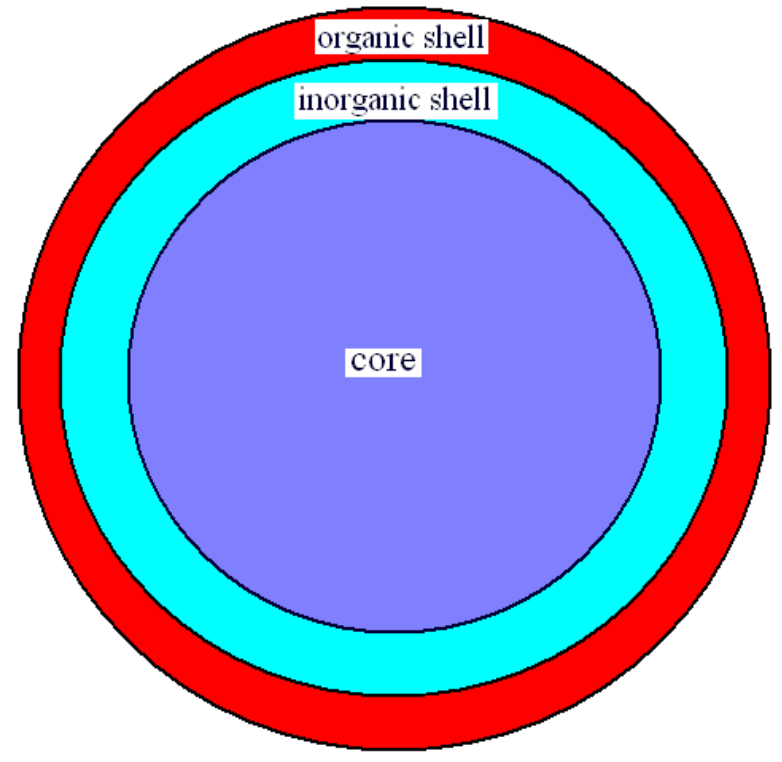

Fig. 2. Schematic representation of the structure of a multi-shell spherical quantum dot. Its core is composed of semiconducting material (its bulk energy gap designate as $E_{g 0}$ ), covered by inorganic shell(s) to passivate the core surface. The outer organic shell ensures that QDs do not aggregate. 


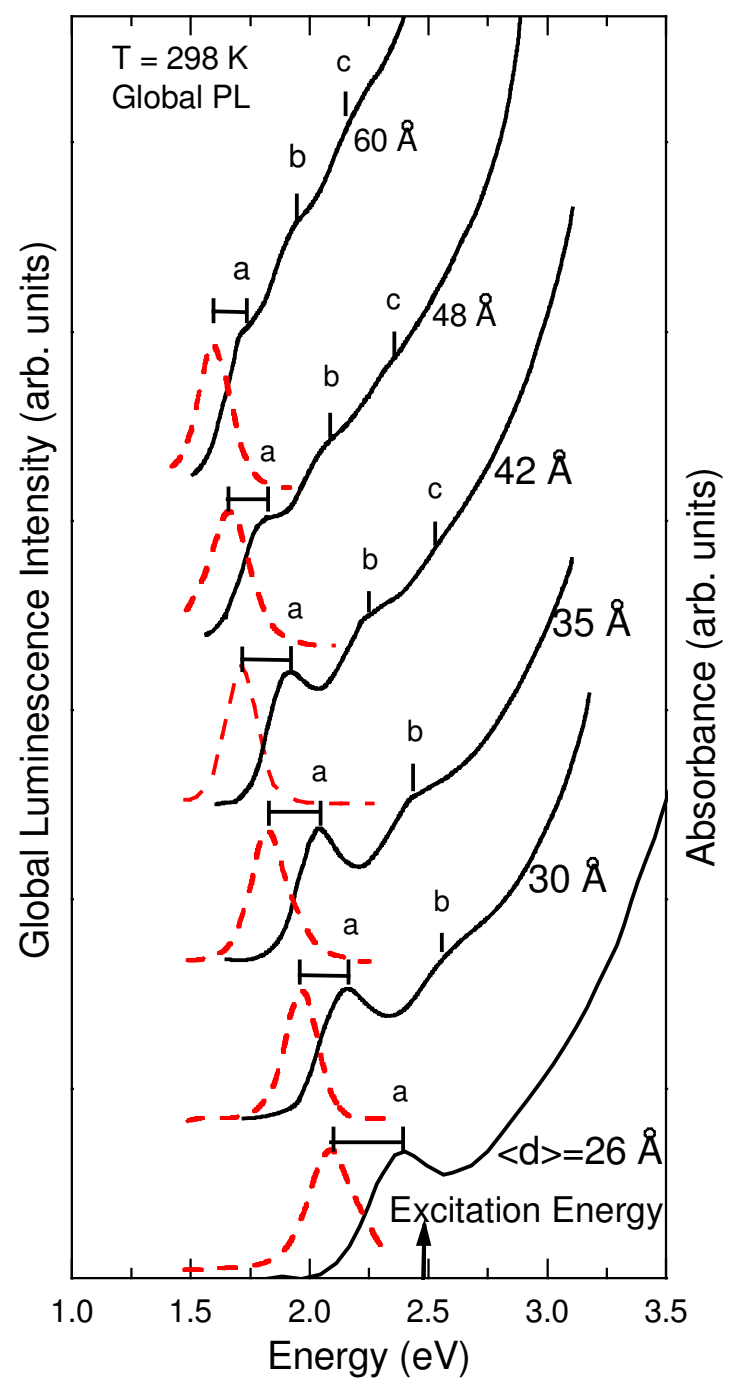

Fig. 3. Absorption (solid line) and global PL (dotted line) spectra at $298 \mathrm{~K}$ for colloidal ensembles of InP QDs with different mean diameters. All QD colloidal samples were photoexcited at $2.48 \mathrm{eV}$. (From the original Fig. 1 in [7].)

$$
I_{P L}\left(E_{p h}\right)=B \int_{0}^{\infty} \frac{1}{r_{i}^{3}} \exp \left(\frac{\left(E_{p h}-E_{P L}\left(r_{i}\right)\right)^{2}}{2 \sigma_{E}^{2}}\right) \exp \left(\frac{\left(r_{i}-\bar{r}\right)^{2}}{2 \sigma_{r}^{2}}\right) d r,(7)
$$

where $B$ is the constant independent of the radius and is determined from the normalization condition of the luminescence intensity $I_{P L}\left(E_{P L}\right)$ to unity, where $E_{P L}\left(r_{i}\right)$ is the energy of the luminescence intensity maximum of QD of radius $r_{i}$.

According to the theory of [8], which treats interaction of semiconductor band states with photonic field, the low energy part of PL spectra is determined by the density of states, while the high energy part of the spectra is defined by the distribution function. The density of states, which contributes to formation of the absorption spectra, is much higher than the density of states, which contributes to formation of luminescence spectrum, therefore, the standard deviation $\sigma_{E}$ for the absorption spectra is larger than that for the luminescence spectra.

\section{Effect of the sizes of quantum dots, their dispersion and thermal broadening the quantized levels on reabsorption}

We estimate the effect of QD size (namely, its core radius), QD size dispersion and the thermal broadening on the reabsorption by using as an example InP QDs. Their absorption and luminescence spectra are shown in Fig. 3. To calculate absorption, formed as a result of the transition of electrons from the level $E_{01}^{h}$ to the level $E_{01}^{e}$, and the intensity of luminescence resulting from transitions of electrons from $E_{01}^{e}$ to $E_{01}^{h}$, we use (6) and (7) and take into account the dependence of the optical bandgap on the QD core radius [9].

To estimate the reabsorption magnitude, the procedure to follow it firstly, using experimentally known energies of the first absorption maximum and the luminescence peak (Fig. 4), we find the mean radius of the quantum dot $\vec{r}$ from Eq. (5). Secondly, using Eqs. (6) and (7), we calculate spectra of absorption and luminescence, normalized to unity. The intersection of these two curves is a measure of reabsorption. It is known that transmittance measurement error does not exceed $0.4 \%$ (see, for example, [10]) and fluorescence intensity measurement error (luminescence) does not exceed $2.6 \%$ (see, e.g., [11, 12]). Therefore, when choosing QDs for solar fluorescent concentrators with minimal reabsorption, we can consider only those semiconductors, in which the value of reabsorption is less than $3 \%$.

In the majority of researches on the efficiency of photovoltaic solar luminescent concentrators, the plastic PMMA plate with QDs is used. Since the plastic plate is transparent in the wavelength range $0.4 \ldots 1 \mu \mathrm{m}$, (or 3.1..1.2 eV), our analysis of the effect of the QD radius and its dispersion on reabsorption was performed for the

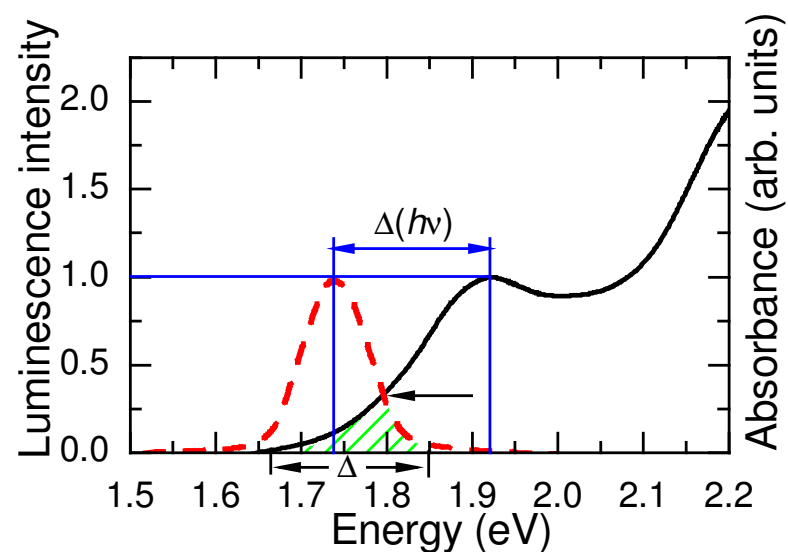

Fig. 4. Spectra of QD absorption (solid line) and luminescence (dashes). The luminescent photons, emitted within the energy range $\Delta$, can be reabsorbed and the hatched area characterize the reabsorption luminescent photons. The energy separation between the first peak of absorption spectrum and the peak of QD luminescence is equal $\Delta h \mathrm{v}$. The QD mean diameter is $3.5 \mathrm{~nm}$. The horizontal arrow indicates the point where the absorption and luminescence spectra are crossing. 
semiconductor QD core with the optical bandgap lying within the range $1.2 \ldots 3.1 \mathrm{eV}$.

Using Eqs. (6) and (7), we find a dependence of nanoparticles reabsorption on their radius and dispersion (10\%, 5\%, 2\% and 1\% QDs dispersion values are considered), and it is shown in Fig. 5. As a distinct feature of the theoretical curves in Fig. 5, the presence of the minimum indicates that the optimum QD core size $r_{\text {min }}$ that minimizes reabsorption can always be chosen. Indeed, QD size increase above $r_{\text {min }}$ reduces the energy separation between the peaks of the absorption and luminescence, thus increasing reabsorption. When QD size decreases below $r_{\min }$, the effect of thermal
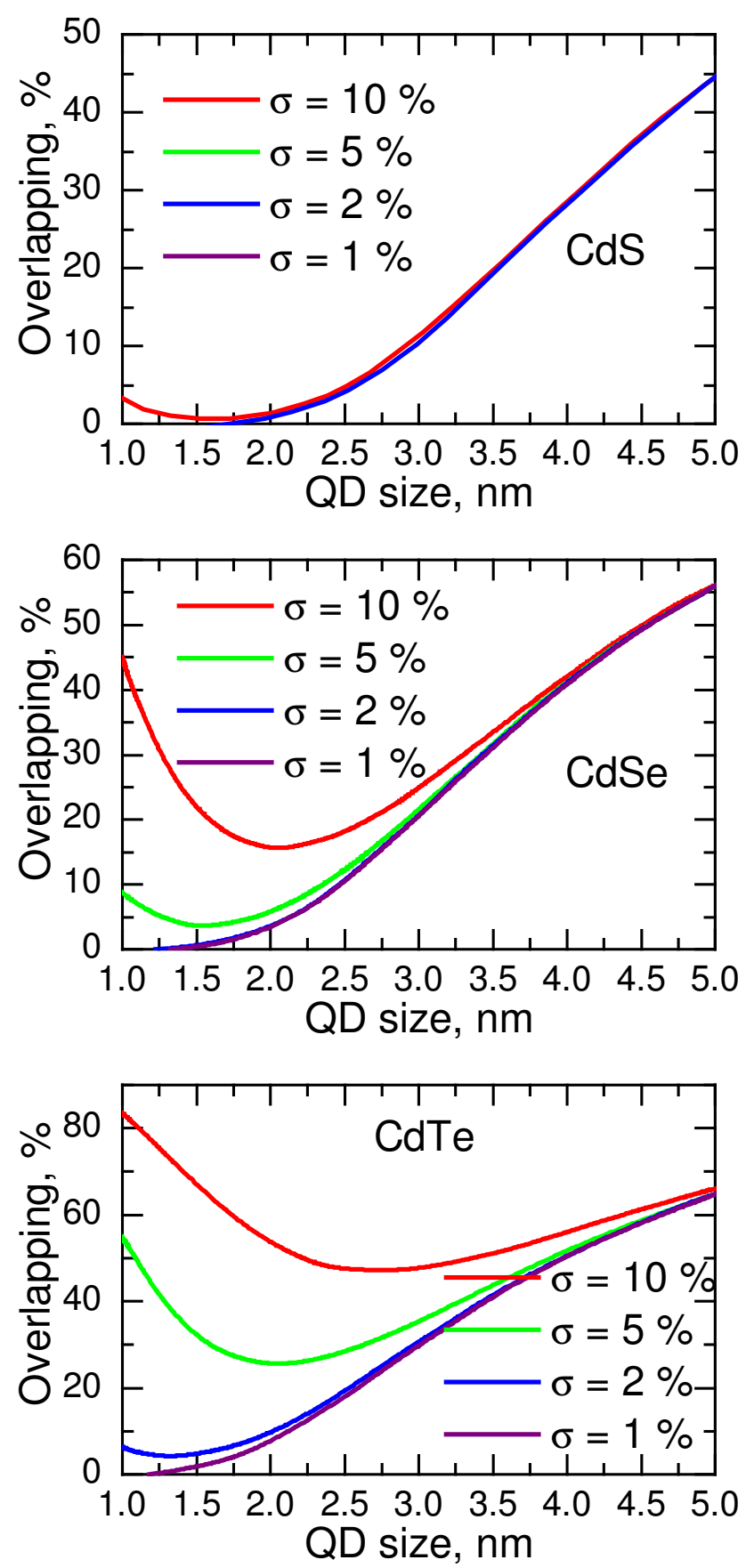

broadening of the quantized energy levels results in the growth of reabsorption. According to Fig. 5, with a decrease of the QD size dispersion, the optimum $r_{\min }$ becomes smaller. It is clear that for CdS-based quantum dots $r_{\min }$ is always above $1.2 \mathrm{~nm}$ at any value of the QD size dispersion. On the other hand, in $\mathrm{PbSe}$ quantum dots even for their core radius of $1 \mathrm{~nm}$ and very low QD size dispersion of $1 \%$, reabsorption cannot be below $54 \%$.

Using the results from Fig. 5 and known semiconductor bulk band gap values, we plot in Fig. 6 the gap dependence of reabsorption for several values of QD core size dispersion, namely: $10 \%, 5 \%, 2 \%$ and $1 \%$. According to Fig. 6, reduction of the QD core size
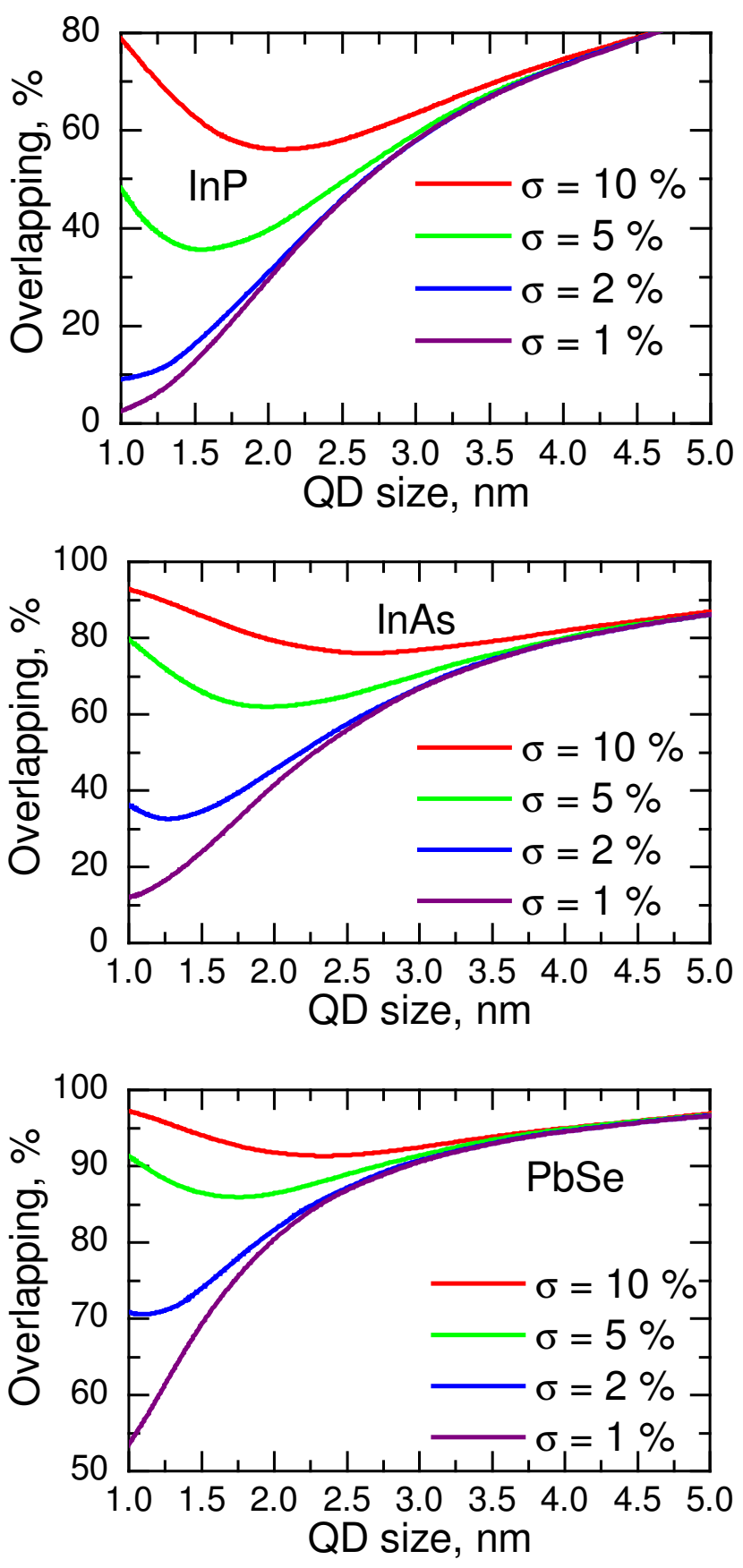

Fig. 5. Dependence of reabsorption on the QD core radius for four values of the size dispersion. To prepare the graphs, experimental dependences of the absorption and luminescence has been considered. The following energy gap values were considered: $\mathrm{CdS}\left(E_{g 0}=\right.$ $2.49 \mathrm{eV}), \mathrm{CdSe}\left(E_{g 0}=1.74 \mathrm{eV}\right), \mathrm{CdTe}\left(E_{g 0}=1.43 \mathrm{eV}\right), \operatorname{InP}\left(E_{g 0}=1.27 \mathrm{eV}\right), \operatorname{InAs}\left(E_{g 0}=0.355 \mathrm{eV}\right), \operatorname{PbSe}\left(E_{g 0}=0.27 \mathrm{eV}\right)$. 


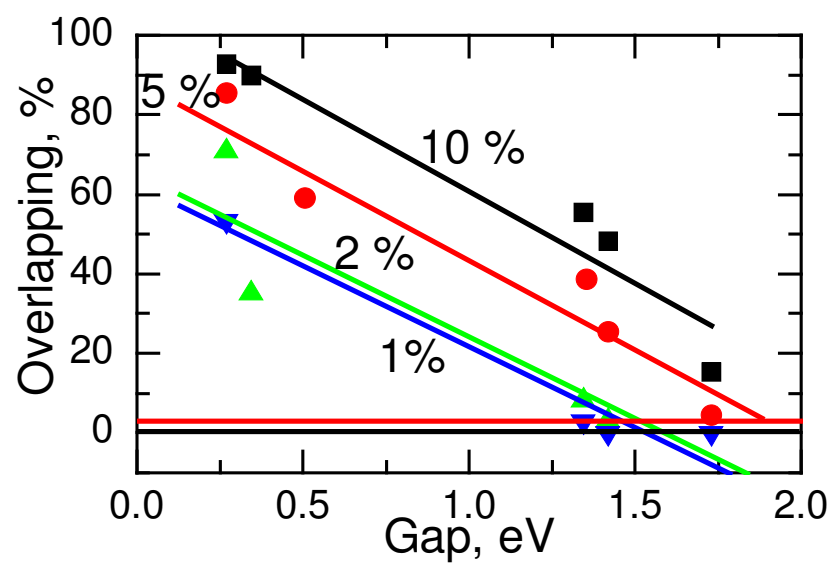

Fig. 6. Dependence of reabsorption losses on the semiconductor band gap $E_{g 0}$ for different size dispersion values. The red horizontal line, close to the $x$-axis, aims to compare the normalized experimental accuracy of the measured QDs spectra to the reabsorption losses.

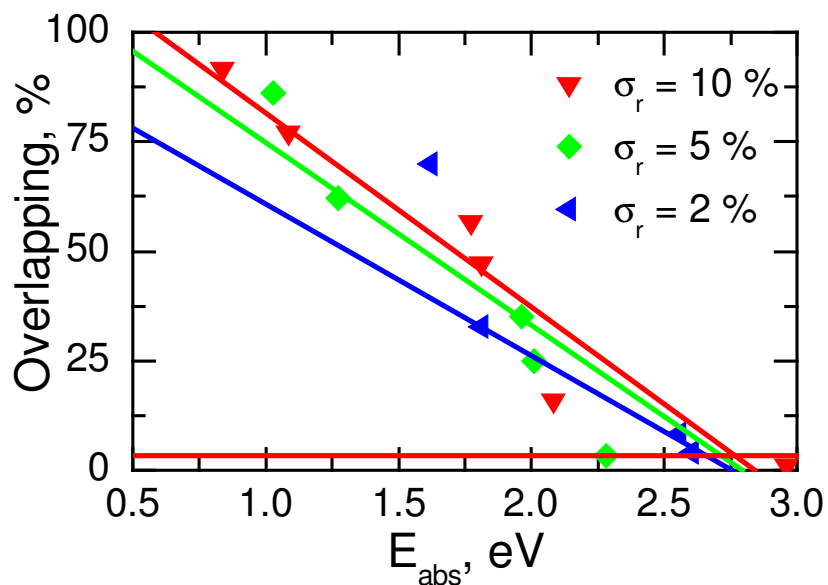

Fig. 7. Dependence of the QD reabsorption on the optical bandgap of the nanoparticle. The red horizontal line, close to the $x$ axis, aims to compare the normalized experimental accuracy of the measured QDs spectra to the reabsorption losses. $T=300{ }^{\circ} \mathrm{C}$.

dispersion results in the parallel shift of the reabsorption dependence on the band gap. Consequently, the value of the band gap, at which the reabsorption becomes negligible, shifts to the lower energy side. Current QD technology offers highly homogeneous nanoparticles with $5 \%$ size dispersion $[8,9]$. At $5 \%$ size dispersion, reabsorption can be negligible (that is below experimental error), if the QD core semiconductor bulk band gap exceeds $1.8 \mathrm{eV}$.

To determine the width of the solar spectrum, from which the incoming photons are transformed into luminescent photons, consider the dependence of QDs reabsorption on the optical band gap. For this, we substitute $r_{\min }$ values from Fig. 5 into Eq. (1) and calculate the optical gap $E_{n, l}^{e, h}$ of the QD core. Alternatively, we can use the gaps, due to the quantum confinement in the nanoparticles, given in [9]. The resulting reabsorption dependence on the optical bandgap of QD is shown in Fig. 7, which demonstrates that the slope of the reabsorption dependence decreases with reduction of the QD size dispersion. It means that with the QD size dispersion decrease, the sunlight absorption bandwidth also decreases. In particular, for the QD cores with $5 \%$ size variation, the range of absorption of solar light photons extends the high-side starting from $2.7 \mathrm{eV}$.

\section{Conclusions}

The quantum dots reabsorption characteristics have been investigated considering their dependence on the QD core size, its dispersion and the bulk band gap of the semiconductor core. It has been shown that with the core size decrease there exists the optimum QD radius that minimizes reabsorption. Decreasing or increasing the size of the QD core with respect to the optimal size always increases reabsorption. It has been found that with decreasing the band gap $E_{g}$ of the core semiconductor (while $E_{g} \geq 1.8 \mathrm{eV}$ ) and $5 \%$ size dispersion, it is possible that reabsorption can be negligibly small (that is, below the experimental detection limit). In contrast, when $E_{g} \leq 1.8 \mathrm{eV}$, further the band gap $E_{g}$ decrease only leads to the growth of reabsorption. For instance, for highly homogeneous with only $1 \%$ size dispersion, $\mathrm{PbSe}$ QDs with $E_{g}=0.27 \mathrm{eV}$, the smallest reabsorption extends to $54 \%$.

\section{References}

1. Kitai A. Luminescent Materials and Applications. John Wiley \& Sons Ltd, The Atrium, Southern Gate, Chichester, West Sussex PO19 8SQ, England.

2. Bera D., Qian L., Tseng T.-K and Holloway P.H. Quantum dots and their multimodal applications: A review. Materials. 2010. 3. P. 2260-2345; doi: 10.3390/ma3042260 file 2679303.

3. Chin P.T-K. Luminescent Properties of Semiconductor Nanocrystals. alexandria.tue.nl>extra2/200811794.pdf.

4. Flogge S. Practical Quantum Mechanics 1. Springer-Verlag, Berlin-New York, 1971.

5. Jasieniak J., Smith L., van Embden J., Mulvaney P., Califano M. Re-examination of the size-dependent absorption properties of CdSe quantum dots. $J$. Phys. Chem. C. 2009. 113. P. 19468-19474.

6. Irman A. Modication of Spontaneous Emission of Quantum Dots by Photonic Crystals. Graduation Thesis, 11 November 2003. Complex Photonic Systems (COPS) Group MESA + Institute Faculty of Science and Technology University of Twente Enschede, The Netherlands cops. nano-cops.com/ sites / default / files / irman.pdf.

7. Micic O.I., Cheong H.M., Fu H., Zunger A., Sprague J.R., Mascarenhas A., and Nozik A.J. Sizedependent spectroscopy of InP quantum dots. $J$. Phys. Chem. B. 1997. 101. P. 4904-4912.

8. van Roosbroeck W. and Shockley W. Photonradiative recombination of electrons and holes in germanium. Phys. Rev. 1954. 94, Issue 6. P. 15581560 . 
9. Baskoutas S., Terzisa A.F. Size-dependent band gap of colloidal quantum dots. J. Appl. Phys. 2006. 99. P. 013708.

10. Ruiz A., Zwinikels J., Bougleux I., Bruce S., Early E. and Barnes P.Y. Interamerican metrology system (Sim 2.2) intercomparison of wavelength scale and photometric scale of spectrophotometry laboratories CENAM-NRC-INMETRO-NIST. SIM 2.2 . Interamerican Metrology System (SIM 2.2).

11. Palmer B.J., Winterton K., Jense M. Scope of accreditation to ISO/IEC 17025:2005. OphirSpiricon LLC 3050 N 300 W North Logan, UT 84431. ANSI-ASQ National Accreditation Board/ACLASS;

www.ophiropt.com/user_files/laser/OphirSpiriconScope.pdf.

12. Firosz K. Scope of accreditation to ISO/IEC 17025:2005. Molecular Devices, Inc. 1311 Orleans Drive Sunnyvale, CA 94089. www.moleculardevices.com/sites/default/files/asset/ document/iso-iec-17025-certification-from-a2la.pdf

\section{Authors and CV}

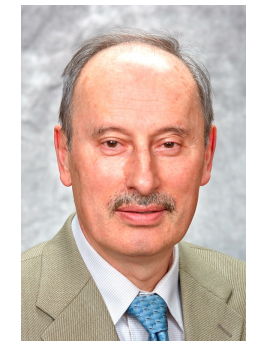

Shkrebtii A.I. Professor, Ph.D. in Physics and Mathematics, Professor at the University of Ontario, Institute of Technology. The area of scientific interests of Prof. Shkrebtii includes solid state physics, semiconductors and their surfaces as well as nanomaterials,

nonlinear optical phenomena, electronic structural and dynamical properties of novel materials.

University of Ontario Institute of Technology

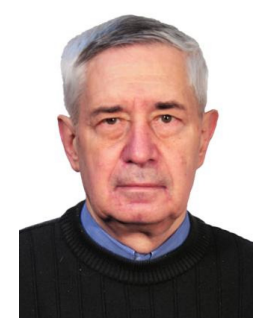

Sachenko A.V.: Professor, Doctor of Sciences in Physics and Mathematics, Chief Researcher at the Department of Semiconductor Surface Physics and Photoelectricity, V. Lashkaryov Institute of Semiconductor Physics, NAS of Ukraine.

The area of scientific interests of Prof. Sachenko includes physics of semiconductors and photovoltaics device simulation.

V. Lashkaryov Institute of Semiconductor Physics, NAS of Ukraine

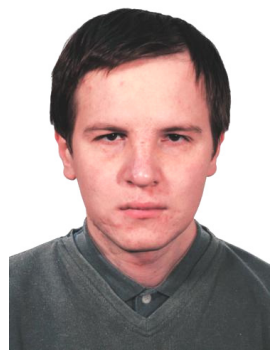

Sokolovskyi I.O.: Ph.D. in Physics and Mathematics, Senior Scientist at the Department of Semiconductor Surface Physics and Photoelectricity, V. Lashkaryov Institute of Semiconductor Physics, NAS of Ukraine. The area of scientific interests of Dr. Sokolovskyi includes physics of photoconversion, analytical and numerical device simulation.

V. Lashkaryov Institute of Semiconductor Physics, NAS of Ukraine

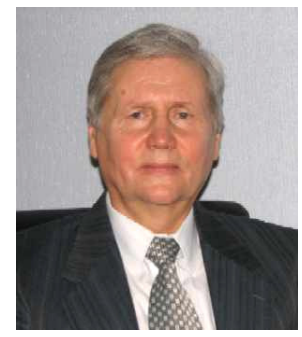

Kostylyov V.P.: Doctor of Sciences in Physics and Mathematics, Head of the Laboratory of Physical and Technical Fundamentals of Semiconductor Photoenergetics, V. Lashkaryov Institute of Semiconductor Physics, NAS of Ukraine. The area of scientific interests of Dr. Kostylyov includes photovoltaic energy conversion physics in semiconductors and semiconductor photoconverters development.

V. Lashkaryov Institute of Semiconductor Physics, NAS of Ukraine

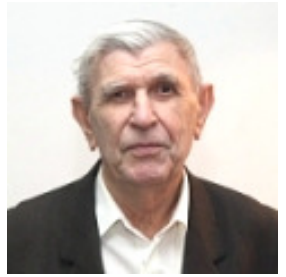

Kulish M.R.: Doctor of Sciences in Physics and Mathematics, Leading Researcher at the Department of Optics and Spectroscopy, V. Lashkaryov Institute of Semiconductor Physics, NAS of Ukraine. The area of scientific interests of Dr. Kulish includes the investigation of optical and nonlinear optical properties of 3D and OD semiconductor structures.

V. Lashkaryov Institute of Semiconductor Physics, NAS of Ukraine 